\title{
Microsurgical treatment of a tentorial galenic dural arteriovenous fistula
}

\author{
Omar Choudhri, MD, and Gary K. Steinberg, MD, PhD \\ Stanford University Medical Center, Department of Neurosurgery, Stanford, California
}

Tentorial dural arteriovenous fistulae (TDAVFs) are complex lesions with the arteriovenous fistula located between the leaves of the tentorium cerebelli. While a large portion of dural arteriovenous fistulae are treated endovascularly, TDAVF may require additional microsurgical treatment given their high risk of hemorrhage and multitude of feeders. We describe the case of a 65-year-old male who presented with hemorrhage from a straight sinus and galenic TDAVF. The straight sinus portion of the fistula was obliterated by 3 endovascular treatments and 1 microsurgical treatment. The galenic component of the TDAVF persisted and was approached via a posterior interhemispheric approach in a lateral position. This video demonstrates surgical technique and anatomy associated with this rarely seen dural arteriovenous fistula.

The video can be found here: https://youtu.be/iOLzWOabLZO.

KEYWORDS galenic fistula; tentorial incisura; supracerebellar infratentorial; microsurgery; dural arteriovenous fistula; tentorial fistula; vein of Galen; video 